\title{
Parâmetros e tendências genéticas para peso ao sobreano de animais Nelore criados no estado do Paraná, Brasil
}

\author{
Parameters and genetic trends for weight at yearling Nellore animals raised in \\ the Parana state, Brazil
}

\begin{abstract}
Júlio Cesar de Souza1 ${ }^{1}$ Marcos Paulo Gonçalves de Resende ${ }^{2}$, Luiz Otavio Campos da Silva ${ }^{3}$, Marco Gusmão ${ }^{4}$, Andrea Gondo ${ }^{3}$, Marcelo Falci Mota ${ }^{4}$, José Antonio Freitas ${ }^{5}$ e Rosana Moreira da Silva ${ }^{6}$

'Universidade Federal de Mato Grosso do Sul. Campo Grande, Mato Grosso do Sul, Brasil. ${ }^{2}$ Universidade Estadual do Sudoeste da Bahia, Jequie, Bahia, Brasil. ${ }^{3}$ Empresa Brasileira de Pesquisa Agropecuária - Gado de Corte, Campo Grande, Mato Grosso do Sul, Brasil. ${ }^{4}$ Médico Veterinário Autônomo. Rondonópolis, Mato Grosso, Brasil.

${ }^{5}$ Universidade Federal da Fronteira Sul, Realeza, Paraná, Brasil. 'Universidade Federal do Paraná, Palotina, Paraná, Brasil.

${ }^{7}$ Universidade Estadual de Mato Grosso do Sul, Aquidauana, Mato Grosso do Sul, Brasil.
\end{abstract}

KEYWORDS:

Beef cattle; heritability; zebu.

\section{PALAVRAS CHAVE:}

Gado de corte; herdabilidade; zebu.

\section{ABSTRACT}

Environmental effects and genetic parameters were estimated for weights at 365 and 550 days for Nellore cattle raised in the Parana State. Least Squared Means methodology, was used to estimate environmental effects. The model included as fixed effects year and month of birth calf, sex and age of dam at calving as a covariate and error as random effects. The variance components were estimated using an animal model considered as fixed effects contemporary group and age of dam. As random effect were composite of direct and maternal genetic additive effects and permanent environment. The genetic trends for direct and maternal effects were estimated by the regression of annual breeding value means on the animals' year of birth. The heritability were $0.24 \pm 0.03$ and $0.35 \pm 0.05$ for direct genetic effect and $0.08 \pm 0.03$ and $0.03 \pm 0.04$ for maternal heritability for weights at 365 and 550 days, respectively. The genetic trends of the direct and maternal effects were $0.506 \mathrm{~kg} / \mathrm{year}$ and $-0.184 \mathrm{~kg} /$ year for weights at 365 days; and $0.712 \mathrm{~kg} / \mathrm{year}$ and $0.187 \mathrm{~kg} / \mathrm{year}$ for weights at 550 days. The results indicate the existence of adequate genetic variability to enable successful selection of breeding stock and realization of genetic gain.

\section{RESUMO}

Objetivou-se avaliar os efeitos de meio e estimar os parâmetros e tendência genética para peso aos 365 e 550 dias de animais da raça Nelore criados no estado do Paraná. Utilizou-se do método dos quadrados mínimos, incluindo os efeitos fixos de ano, mês de nascimento, sexo e a co-variável idade da vaca ao parto (linear e quadrática). Os parâmetros genéticos foram estimados utilizando-se um modelo animal que continha os efeitos aleatórios genéticos direto e materno, de ambiente permanente e o erro; além do efeito fixo de grupo contemporâneo e da co-variável idade da vaca ao parto. A tendência genética foi obtida por meio de análise de regressão linear do valor genético do animal sobre o ano de nascimento. As herdabilidades diretas foram 0,24 $\pm 0,03$ e 0,35 $\pm 0,05$; as herdabilidades maternas foram $0,08 \pm 0,03$ e 0,03 $\pm 0,04$, para peso aos 365 e 550 dias, respectivamente. As tendências genéticas direta e materna foram 0,506 kg/ano e -0,184 kg/ano para P365; e 0,712 kg/ano e 0,187 kg/ano para P550. Os resultados indicam a existência de variabilidade genética o que possibilitam ganhos através de seleção. 


\section{INTRODUÇÃO}

O Brasil é um país de grande extensão territorial, sendo a atividade pecuária bastante intensa e importante geradora de renda na balança comercial. O uso de programas de melhoramento genéticos específicos, abrangendo os diferentes sistemas de produção é importante e as estimativas de parâmetros genéticos e estimativas de efeito de meio contribuem na realização de ajustes dos pesos às respectivas idades, além de que, possibilitam avaliar as tendências e ganho genético dos rebanhos ao longo de um determinado período.

Diversos trabalhos envolvendo efeitos ambientais como ano e estação de nascimento do bezerro, sexo, fazenda e idade da vaca foram reportados por SOUZA et al. (2000), SOUZA et al. (2002), GUIMARÃES et al. (2003), TORAL et al. (2004). Estudando herdabilidade direta e materna, e tendência genética, SOUZA et al. (2005) e FERRAZ FILHO et al. (2002) mostram valores de média a alta magnitude. Quanto ao progresso genético, SANTOS et al. (2012) reportam a necessidade de avaliar as tendências genéticas, fenotípicas e de ambiente para características de importância econômica, e utilizar essas respostas como elementos norteadores de ações futuras para melhorar a produção animal.

LAUREANO et al. (2011) estudando tendência genética de animais da Raça Nelore, reportaram 0,219 kg de ganho para características ao sobreano, com um incremento de $0,08 \%$ nas médias anuais. FERRAZ FILHO et al. (2002), que relataram tendências genéticas diretas iguais $0,280 \mathrm{~kg} / \mathrm{ano}$, respectivamente, para peso ao sobreano, as quais corresponderam a incrementos na mudança genética anual de $0,10 \%$ nas médias dos pesos, para animais da raça Tabapuã.

MUCARli y OLIVEIRA (2003) avaliando animais de raça zebuína, relataram tendências genéticas de $0,345 \mathrm{~kg} /$ ano, para peso ao sobreano. Segundo os valores revelados, com ganhos genéticos relativamente baixos, podem estar associados à baixa variabilidade genética observada nos rebanhos avaliados (LAUREANO et al., 2011). Nesse sentido, objetivou-se avaliar os efeitos de meio, estimar parâmetros genéticos e as tendências genéticas para os pesos aos 365 e 550 dias de bovinos da raça Nelore criados no estado do Paraná.

\section{MATERIAL E MÉTODOS}

Utilizaram-se informações de animais da raça Nelore, criados no estado do Paraná, pertencentes ao banco de dados da Associação Brasileira de Criadores de Zebu e do Centro Nacional de Pesquisa de Gado de Corte da EMBRAPA (Empresa Brasileira de Pesquisa Agropecuaria). Para estimativas dos efeitos de meio, as análises foram realizadas utilizando-se o método dos quadrados mínimos, para dados desbalanceados. Considerou-se para avaliação dos efeitos ambientais para o peso aos $365(n=13.218)$ e 550 dias de idade ( $n=8.007$ ) informações obtidas no período de 1980 a 2000, referente a 530 touros distribuídos em 182 fazendas.

Incluiu-se no modelo estatístico os efeitos fixos de ano e mês de nascimento do bezerro, sexo, idade da vaca ao parto (linear e quadrático como covariável), além do efeito aleatório do touro dentro de fazenda (nem todos os touros trabalharam em todas as fazendas) e do erro, conforme descrito a seguir:

$Y_{i j k m n}=\mu+T(F)_{j}+A_{k}+M_{l}+S_{m}+b_{1}\left(X_{i j k m}-\bar{X}\right)+b_{2}\left(X_{j k l m}-\bar{X}\right)^{2}+e_{i j k l m n}$,

Em que:

$\mathrm{Y}_{\mathrm{ijklmn}}=$ variável dependente (P365 ou P550);

$\mu=$ média geral para a característica avaliada;

$\mathrm{TF}_{\mathrm{ij}}=$ efeito aleatório do reprodutor i dentro da fazenda j;

$A_{k}=$ efeito fixo do ano de nascimento k;

$\mathrm{M}_{\mathrm{l}}=$ efeito fixo do mês I de nascimento; $\mathrm{S}_{\mathrm{m}}=$ efeito fixo do sexo da cria m;

$\mathrm{X}_{\mathrm{ijklm}}=$ idade da vaca ao parto;

$\mathrm{b}_{1}=$ coeficiente de regressão para idade linear;

$\mathrm{b}_{2}=$ coeficiente de regressão para idade quadrática;

$\mathrm{e}_{\mathrm{ijk} \mid \mathrm{mn}}=$ erro aleatório, normal e independentemente distribuído com média zero e variância $\sigma^{2}\left(\operatorname{NID}\left(0, \sigma^{2}\right)\right)$.

Para estimativa dos componentes de variância e covariâncias, assim como dos parâmetros para efeitos genéticos diretos e maternos, os números de animais utilizados foram 19.150 e 11.190, para P365 e P550, respectivamente. Os grupos contemporâneos foram formados levando-se em consideração os efeitos de mês e ano de nascimento dos bezerros, sexo e fazenda, combinados. A metodologia utilizada para obter as estimativas foi a da máxima verossimilhança restrita, programa MTDFREML, de BOLDMAN et al., (1995), com o seguinte modelo:

$Y=X \beta+Z a+M m+l p+e$,

Em que $Y=$ vetor das informações; $X=$ matriz de incidência dos efeitos fixos; $\beta=$ vetor dos efeitos fixos de grupo contemporâneo e idade da vaca ao parto; $Z$ = matriz de incidência para os efeitos aleatórios genéticos diretos de animal; a = vetor dos efeitos aleatórios genéticos diretos de animal; $M=$ matriz de incidência para os efeitos aleatórios genéticos maternos de animal; $\mathrm{m}=$ vetor de efeitos genéticos maternos de animal; $\mathrm{I}=$ matriz de incidência do efeito do ambiente permanente; $p=$ vetor dos efeitos de ambiente permanente e, $\mathrm{e}=$ vetor dos erros aleatórios associados a cada observação.

As tendências genéticas para os efeitos aditivos diretos e maternos foram estimadas pela regressão ponderada 
das médias anuais dos valores genéticos diretos $\mathrm{e}$ maternos sobre o ano de nascimento do animal.

\section{RESULTADOS E DISCUSSÃO}

Todas as fontes de variação do modelo influenciaram $(P<0,0001)$ as características estudadas (Tabela 1), evidenciando a sua importância ao se fazer comparações de pesos e também para a escolha de reprodutores. A média ajustada e o erro padrão para os 365 dias de idade foram $267,0 \pm 2,6 \mathrm{~kg}$, com $\mathrm{R}^{2}$ de $62,7 \%$ e o coeficiente de variação de $17,2 \%$. Para o peso aos 550 dias, a média e o erro padrão foram $359,0 \pm 3,97 \mathrm{~kg}$, com um $R^{2}$ de $69 \%$ e coeficiente de variação de $16,96 \%$. $O$ efeito de mês de nascimento, também significativo $(P<0,0001)$ para ambos os pesos e revelou que deve haver variações nas condições edafoclimáticas e que estas influenciam diretamente sobre as características produtivas dos animais, principalmente quando se trata de animais mantidos em pastagem.

Tabela 1. Resumo da análise de variância para os pesos aos 365 e 550 dias de idade criados no estado do Paraná.

\begin{tabular}{|c|c|c|c|c|}
\hline \multirow[t]{2}{*}{$\begin{array}{c}\text { Fontes de } \\
\text { Variação }\end{array}$} & \multicolumn{2}{|c|}{ Peso aos 365 dias } & \multicolumn{2}{|c|}{$\begin{array}{c}\text { Peso aos } 550 \\
\text { dias }\end{array}$} \\
\hline & GL & QM & GL & QM \\
\hline Touro (Fazenda) & 2.206 & $8771,09^{* * *}$ & 1677 & $13517,34^{* * *}$ \\
\hline Mês & 11 & $39695,76^{* * *}$ & 11 & $40112,15^{\star * *}$ \\
\hline Ano & 20 & $14132,58^{* * *}$ & 20 & $23413,41^{* \star *}$ \\
\hline Sexo & 1 & $4967855,25^{\star \star \star}$ & 1 & $3016,95^{\star * *}$ \\
\hline Idade da vaca linear & 1 & $110140,22^{* * *}$ & 1 & $55335,57^{* * *}$ \\
\hline $\begin{array}{l}\text { Idade da vaca } \\
\text { quadratica }\end{array}$ & 1 & $123059,90^{* * *}$ & 1 & $53734,29^{\star * *}$ \\
\hline Erro & 10977 & & 6295 & \\
\hline Total & 13.217 & & 8.006 & \\
\hline
\end{tabular}

Os animais mais pesados aos 365 dias foram os nascidos no mês de maio, $278,1 \pm 2,9 \mathrm{~kg}$, e os mais leves, com peso de 254,8 $\pm 2,6 \mathrm{~kg}$, nasceram no mês de janeiro (Figura 1). Para o peso aos 550 dias, o mês de nascimento em que ocorreu a maior média foi o mês de julho, com $373,1 \pm 4,0$ $\mathrm{kg}$ e o de menor média foi o mês de abril com $344,6 \pm 4,2$ $\mathrm{kg}$. Para o peso aos 550 dias, os animais mais pesados nasceram no mês de julho, $373,1 \pm 3,97 \mathrm{~kg}$ e os mais leves, nascidos em abril, pesaram $344,6 \pm 4,2 \mathrm{~kg}$.

Tais evidências orienta a implantação de uma estação de monta com intuito de obter bezerros mais pesados a essas idades, no início de maio e término em setembro. Ressaltase a necessidade de planejamento nutricional para as matrizes e, até mesmo suplementação com volumoso e ou concentrado, para obter-se balanço energético positivo, garantindo a ciclicidade ovariana, visto baixa produção de forragens tanto quantitativamente quanto qualitativamente neste período (seca).

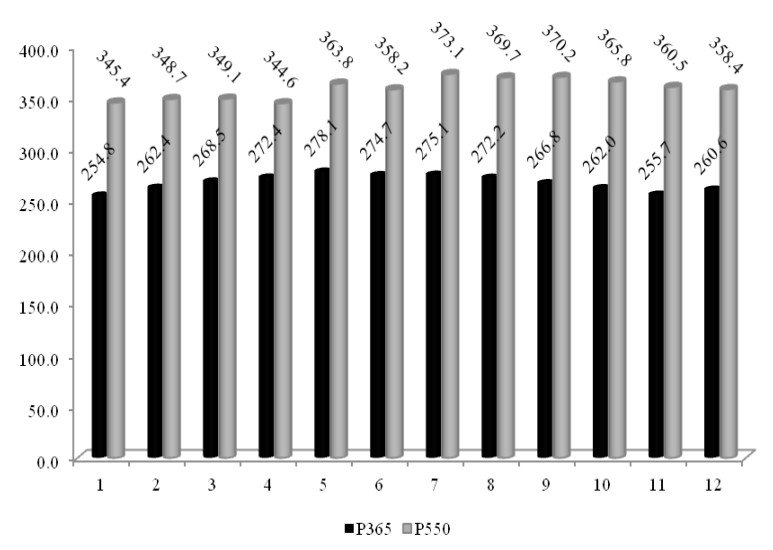

Figura 1. Peso $(\mathrm{kg})$ aos 365 e 550 dias de idade conforme o mês de nascimento, para animais da raça Nelore, criados no estado do Paraná.

$O$ efeito de ano de nascimento foi significativo $(P<0,0001)$ mostrando as variações climáticas ao longo dos anos, devido a efeitos sazonais de chuvas ou longos períodos de estiagens, presença e/ou intensidade de geada, etc. Dessa forma, em anos com alta precipitação propiciando um aumento da produção de forragem e conseqüentemente um maior ganho de peso dos animais. Quando a estação da seca é severa, a produção de forragem fica prejudicada e o ganho de peso fica comprometido.

Durante o período avaliado os animais nascidos no ano 2000, tanto para peso aos 365 como aos 550 dias de idade, apresentaram pesos mais elevados, $288,48 \pm 5,11$ $\mathrm{kg}$ e $396,07 \pm 9,55 \mathrm{~kg}$, respectivamente. Os menores pesos observados foram os obtidos no ano de 1981 para o peso aos 365 dias e no ano de 1982 para peso aos 550 dias de idade, com $242,08 \pm 6,72 \mathrm{~kg}$ e $337,31 \pm 6,60$ $\mathrm{kg}$, respectivamente.

$O$ efeito do sexo dos animais também foi significativo $(P<0,0001)$ para as duas características avaliadas. $O$ peso dos machos $(n=6.784$ ) foi $289,30 \pm 2,10 \mathrm{~kg}$ e o das fêmeas $(n=6.434), 244,61 \pm 2,08 \mathrm{~kg}$ aos 365 dias de idade, $15,5 \%$ mais alto nos machos. Para o peso aos 550 dias, os machos $(n=3.790)$ pesaram $400,89 \pm 3,12$ $\mathrm{kg}$ e as fêmeas $(n=4.217) 317,02 \pm 3,05 \mathrm{~kg}$, uma superioridade de $20,9 \%$.

Para peso aos 550 dias, os valores foram $263,23 \pm 46,01$ $\mathrm{kg}$ e $304,18 \pm 52,80 \mathrm{~kg}$ no estado do Mato Grosso do Sul. Observou-se também efeito linear e quadrático da idade da vaca sobre o peso dos animais. A média de idade das matrizes para o peso aos 365 dias foi de $89,5 \pm 41,62$ meses e para peso aos 550 dias, foi de $88,24 \pm 42,09$ meses. Observou-se que as matrizes apresentaram seu ponto máximo (média de idade em que as matrizes produziram animais mais pesados) 
foi aos 103,74 meses para P365 e 107,37 meses para $P 550$, sendo que as vacas no início (jovens) e no final da sua vida produtiva (depois dos 174 meses) produziram progênies mais leves (Figura 2).

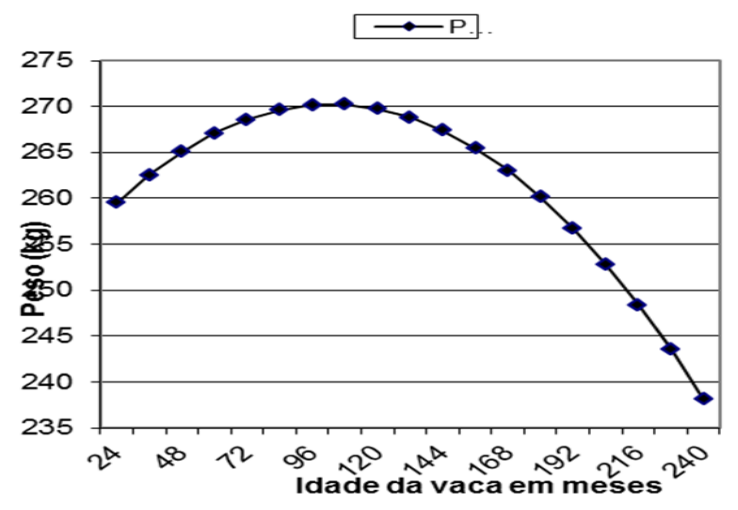

$\longrightarrow P$.

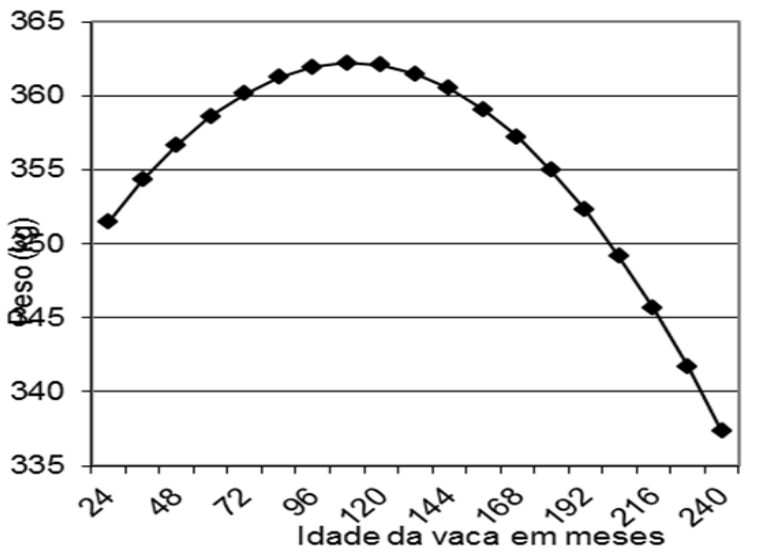

Figura 2. Peso médio aos 365 e 550 dias de idade de animais da raça Nelore, de acordo com a idade da vaca ao parto.

Com base nessas observações, pode-se realizar um descarte seletivo de $10 \%$ ao ano, eliminando as vacas com idade avançada e manter no rebanho as vacas jovens. Esses resultados confirmam os obtidos por SOUZA et al. (2000, que relataram apenas efeito quadrático da idade da vaca sobre peso aos 365 e aos 550 dias de idade. Por outro lado, SOUZA et al. (2002) não verificaram efeito significativo da idade da vaca efeito linear e quadrático sobre o peso aos 365 dias.

Os componentes aditivos diretos foram elevados mostrando variabilidade da característica estudada. As estimativas obtidas para herdabilidade direta e materna para P365 foram 0,24 $\pm 0,03$ e $0,08 \pm 0,03$ e para $\mathrm{P} 550$, foram 0,35 $\pm 0,05$ e 0,03 $\pm 0,04$ respectivamente (Tabela 2). TORAL et al. (2004) obtiveram herdabilidade direta maior para P365 $(0,40)$ e para P550 $(0,35)$. Na tabela 2 pode se observar que os valores das correlações entre os efeitos direto materno foram elevados e negativos para $\mathrm{P} 550$, porém não significativo, visto o valor do desvio-padrão estimado.
Tabela 2. Parâmetros genéticos estimados para os pesos de animais da raça Nelore, criados no estado do Paraná aos 365 (P365) e 550 (P550) dias de idade.

Parâmetro

Variância aditiva direta $\left(\sigma_{\mathrm{a}}^{2}\right)$

Variância matema $\left(\sigma_{m}^{2}\right)$

Co-variância direta matema $\left(\sigma_{a m}\right)$

Variância efeito permanente matemo $\left(\sigma_{\mathrm{pe}}^{2}\right)$

Variância residual $\left(\sigma_{\mathrm{e}}^{2}\right)$

Variância fenotípica $\left(\sigma_{\mathrm{p}}^{2}\right)$

Herdabilidade direta $\left(\mathrm{h}_{\delta}^{2}\right)$

Herdabilidade matema $\left(\mathrm{h}_{\mathrm{m}}^{2}\right)$

Correlação direta materna $\left(r_{\mathrm{dm}}\right)$

Efeito de ambiente permanente $\left(c^{2}\right)$

Proporção ambiental da variância (e1)

\begin{tabular}{cc}
\multicolumn{2}{c}{ Característica } \\
P365 & P550 \\
330,78 & 591,02 \\
104,34 & 50,35 \\
$-123,02$ & $-157,83$ \\
127,65 & 93,92 \\
914,83 & $1.096,58$ \\
$1.354,58$ & $1.674,05$ \\
$0,24 \pm 0,03$ & $0,35 \pm 0,05$ \\
$0,08 \pm 0,03$ & $0,03 \pm 0,04$ \\
$-0,66 \pm 0,12$ & $-0,91 \pm 0,44$ \\
$0,09 \pm 0,02$ & $0,06 \pm 0,03$ \\
$0,68 \pm 0,02$ & $0,66 \pm 0,04$
\end{tabular}

Os valores obtidos para o efeito materno foram baixos, a essas nestas idades, os animais ja encontram-se independente de suas mães, no entanto, o efeito foi negativo. O ganho genético total no período para P365 no período de 1981 a 2000 foi $9,14 \mathrm{~kg}$, com uma equação de ajuste igual á $\mathrm{y}=0.4571 \mathrm{x}-2.4416$ e um $\mathrm{R}^{2}=0.8563$. Para o P550, o ganho genético total foi de $11,96 \mathrm{~kg}$ e a equação de ajuste linear foi $y=0.5978 x$ 3.7309 e um $\mathrm{R}^{2}=0.7708$.

SOUZA et al. (2004) avaliando a tendência genética para o peso aos 205 dias de idade para animais da raça Nelore nas regiões do Pantanal, constataram um ganho de 6,92 kg e para a região do Alto Taquari Bolsão um ganho de $8,13 \mathrm{~kg}$ no período de 1976 a 2001 . FERRAZ FILHO et al. (2002) ao estudarem bovinos da raça Tabapuã, verificaram tendências genéticas do efeito direto de 0,207 e 0,276 kg/ano para peso aos 365 e 550 dias respectivamente.

Apesar de a habilidade materna ser mais importante na fase inicial da vida do animal, bezerros filhos de vacas com maior habilidade materna tendem a desmamar mais pesados e consequentemente são mais pesados nas idades subseqüentes. Verifica-se (Figura 3) que com o aumento do ganho genético direto há uma redução no ganho materno, com intensidade menos acentuada.

A tendência materna no período foi negativa, com perda de $0,188 \mathrm{~kg} / \mathrm{ano}$. No entanto, deve se trabalhar no sentido de ampliar o ganho genético/ano, visto que os animais apresentam potencial genético para as duas características. Malhado et. al. (2010) estudando melhoramento genético e estrutura populacional da Raça Nelore na Região Norte do Brasil, verificaram ganho genético de $1.559 \mathrm{~kg}$ para P365 e $1.951 \mathrm{~kg}$ para P365 e consideraram que para essas duas características houve ganho ao longo do tempo. 

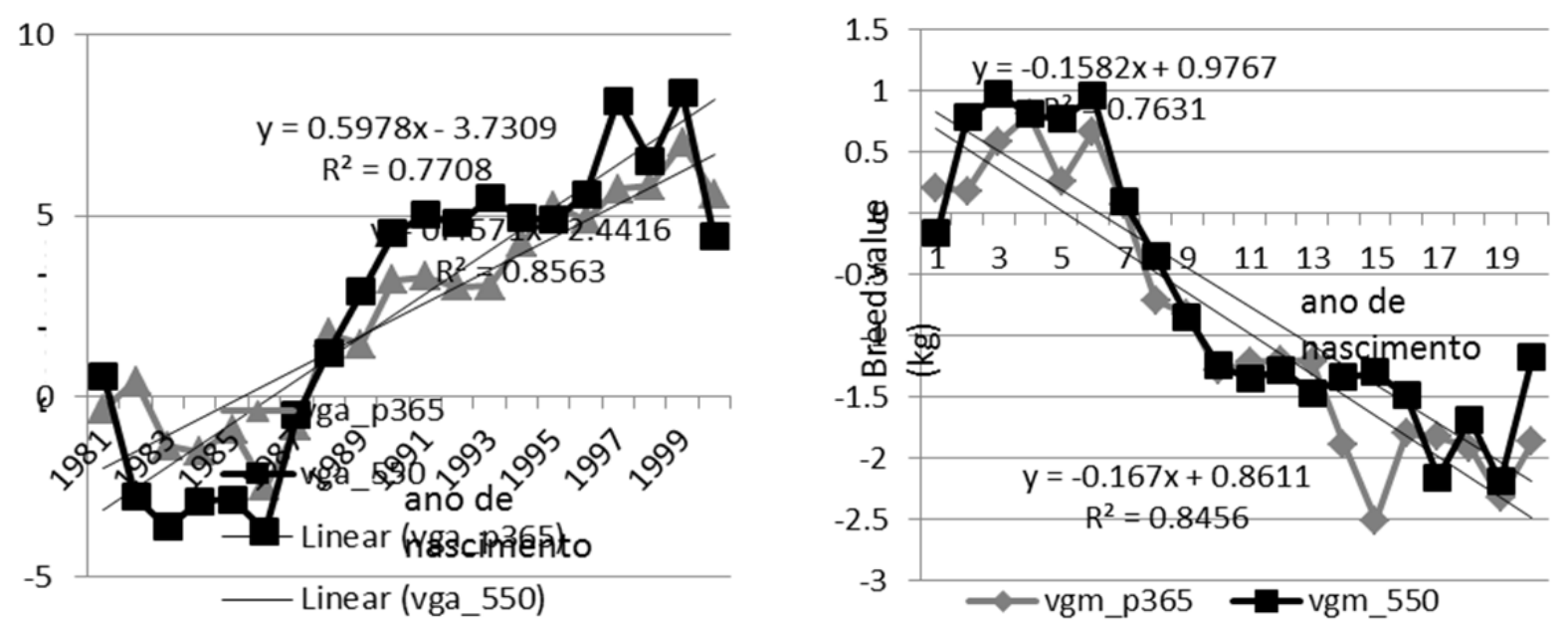

Figura 3. Tendência genética direta (vga = observada; vga_a = estimada) e materna (vgm = observada; vgm_a = estimada) para o p365 e p550.

\section{Conclusões}

Os fatores ambientais exerceram efeito significativo sobre as características de P365 e P550 dias, portanto, é necessário levá-los em consideração quando da seleção em gado de corte. Os coeficientes de herdabilidade estimados indicam possibilidade de se realizar seleção baseando-se nos pesos a essas idades. Os animais criados no Estado do Paraná apresentaram ganho genético no período estudado, mas com perdas quanto aos efeitos maternos.

\section{REFERÊNCIAS}

BOLDMAN, K.G.; KRIESE, L.A.; VAN VLECK, L.D.; VAN TASSEL, C.P.; KACHMAN, D. 1995. A manual for use of MTDFREML. A set of programs to obtain estimates of variances and covariances. ARS, USDA, Washington, DC.

FERRAZ FILHO, P.B.; RAMOS, A.A.; SSILVA, L.O.C.; SOUZA, J.C.; ALENCAR, M.M.; MALHADO, C.H.M. 2002. Tendência genética dos efeitos direto e materno sobre os pesos à desmama e pós-desmama de bovinos da raça Tabapuã no Brasil. Revista Brasileira de Zootecnia 31(1): 635-640.

GUIMARÃES, L.B.; FERRAZ FILHO, P.B.; SOUZA, J.C.; SILVA, L.O.C. 2003. Aspectos genéticos e de ambiente sobre pesos pré e pós desmama em bovinos da raça Tabapuã na região pecuária oeste São Paulo - Paraná. Archives of Veterinary Science 8(1):109-119.

LAUREANO, M.M.M.; BOLIGON, A.A.; COSTA, R.B.; FORNI, S.; SEVERO, J.L.P; ALBUQUERQUE, L.G. 2011. Estimativas de herdabilidade e tendências genéticas para características de crescimento e reprodutivas em bovinos da raça Nelore. Arquivos Brasileiro de Medicina Veterinária e Zootecnia 63(1): 143-152.

MALHADO, C.H.M; CARNEIRO, P.L.S.; MALHADO, A.C.M.; MARTINS FILHO, R.; BOZZI, R; LADLE, R.J. 2010. Genetic improvement and population structure of the Nellore breed in northern Brazil. Pesquisa Agropecuária Brasileira, Brasília 45(10):1109-1116.

MUCARI, T.B.; OLIVEIRA, J.A. 2003. Análise genético-quantitativa de pesos aos 8, 12, 18 e 24 meses de idade em um rebanho da raça Guzerá. Revista Brasileira de Zootecnia 32(1):1604-1613. 
SANTOS, L.H.; OLIVEIRA, S.M.P.; MALHADO, C.H.M.; CARNEIRO, P.L.S.; MARTINS FILHO, R.; LOBO, R.N.B.; RODRIGUES, D.S. 2012. Estrutura populacional e tendências genéticas e fenotípicas da raça Guzerá no Nordeste do Brasil. Revista Brasileira de Saúde e Produção Animal 13(4):1032-1043.

SOUZA, J.C.; RAMOS, A.A.; SILVA, L.O.C.; FILHO, K.E.; ALENCAR, M.M.; WECHSLER, F.S.; FILHO, P.B.F. 2000. Fatores do ambiente sobre o peso ao desmame de bezerros da raça Nelore em regiões tropicais brasileiras. Ciência Rural 30(5):881-885.

SOUZA, J.C.; MALHADO, C.H.M.; SILVA, L.O.C.; FERRAZ FILHO, P.B. 2002. Efeito do ambiente sobre o peso de bovinos da raça Guzerá no estado de São Paulo. Archives of Veterinary Science 7(1):57-63.

TORAL, F.L.B.; SILVA, L.O.C.; MARTINS, E.N.; GONDO, A.; SIMONELLI, S. M. 2004. Interação Genótipo x Ambiente em Características de Crescimento de Bovinos da Raça Nelore no Mato Grosso do Sul. Revista Brasileira Zootecnia 33(6):1445-1455. 